\title{
Non-targeted direct infusion soluble organic analysis on Tarda meteorite extract
}

\author{
JUNKO ISA $^{1}$, FRANÇOIS-RÉGIS ORTHOUS-DAUNAY ${ }^{1}$, \\ VÉRONIQUE VUITTON $^{1}$, LAURÈNE FLANDINET ${ }^{1}$ AND \\ MICHAEL E. ZOLENSKY ${ }^{2}$ \\ ${ }^{1}$ Université Grenoble Alpes CNRS/IPAG \\ ${ }^{2}$ NASA Johnson Space Center \\ Presenting Author: 13junko@gmail.com
}

The differences in chemistry, mineralogy, and structure of chondrites demonstrate the variations in time/space of their forming regions in the solar nebula. In contrast, the variation of the organic reserves in the solar nebula is unclear. Furthermore, the subsequent evolution of organic matter taking place on their parent body is still under debate. In a previous study on Tagish Lake (C2 ungrouped), we showed that the primordial soluble organic matter (SOM) could be distinguished from the parent body's secondary alteration through mass distribution. This study will show the Tarda meteorite SOM primordial distribution by using the model from our previous study. We discuss its organic reservoirs and secondary alteration effects. We also compare the character of the SOM in Tarda with the previously analysed SOM found in Orgueil (CI).

Sample and Method: Tarda (fall, 2020) was classified as a C2 ungrouped carbonaceous chondrite. Its bulk three oxygen isotope composition is distinct from other chondrite groups but similar to CI-chondrite and Yamato-type values [1]. We analysed the methanol/toluene extract by utilizing the high-resolution Orbitrap mass spectrometer located at the Institute de Planétologie et d'Astrophysique Grenoble (IPAG) by directly injecting and ionizing the SOM and its solvent by using the Heated Electrospray Ionization (HESI) source. After spectra acquisition, post-processing data analysis tools with the name Attributor [2], which were developed in-house, were employed.

Preliminary Results: The Tarda SOM mass distribution was well fit by using the model interpreted as the primordial SOM distribution in Tagish Lake. This match between the two distribution patterns implies the presence of a common organic reservoir or a common mechanism for SOM formation. The Orgueil mass spectrum shows a more complex mass distribution and that seems to be affected by secondary alteration processes. In contrast to the mass-distribution, SOM's elemental abundance was distinct. The Tarda extract was enriched in S-bearing organic compounds which contrasts with the Tagish Lake extract consisting of mostly CHNO elements. The presentation will also discuss the application of our technique to Ryugu and Bennu samples.

[1] Meteoritical bulletin database, [2] Orthous-Daunay, F. R. et al. (2019) Geochemical Journal, 53(1), 21-32. 